\title{
Adaptación, validación y estandarización del Mini Cuestionario de la Organización Personal en población universitaria colombiana ${ }^{4}$
}

\author{
Diego Alfredo Tamayo Lopera \\ Magister en Neuropsicología \\ Institución Universitaria de Envigado, Colombia \\ Correo electrónico: pditalo@gmail.com
}

Alejandro León Uribe Magíster en Terapia Cognitiva Posracionalista CETEPO Centro de Terapia Posracionalista Correo electrónico: alejo.leon@gmail.com

Jonathan Andrés Hernández Calle Magister en Epidemiología Institución Universitaria de Envigado, Colombia Correo electrónico: nathancalle@yahoo.es
Recibido: 12/07/2018

Evaluado: 15/01/2019

Aceptado: 14/05/2019

\section{Resumen}

El MCOP es un cuestionario auto-aplicado de origen italiano para valorar la Organización del Significado Personal (OSP), constructo teórico de la personalidad fundamental para la psicoterapia cognitiva posracionalista, creada por Vittorio Guidano. El presente artículo es una adaptación y validación de dicho instrumento en población colombiana. Método: La adaptación y validación se llevó a cabo aplicando el instrumento a una muestra de 243 estudiantes universitarios. Resultados: Se conformaron 4 factores principales como en la versión original del test, cuyos índices de consistencia interna medidos mediante el Alfa de Cronbach oscilaron entre .57 y .67, con una consistencia interna total del test de .74. En el análisis de los factores según el sexo se encontró una diferencia estadísticamente significativa en las OSP Depresiva ( $p=.043$ ) y OSP Fóbica $(p=.045)$, mostrando los hombres mayor tendencia a presentar la primera y las mujeres la segunda organización. Conclusión: El MCOP traducido al español evidenció una buena consistencia interna, lo que permite ser usado en investigaciones científicas en el contexto universitario colombiano.

Keywords

Content validity; rumors; Lawshe's Method; Exploratory Factor Analysis; Likert Scale

4 Para citar este artículo: Tamayo, D.A., León, A., Hernández, J. A. (2020). Adaptación, validación y estandarización del Mini Cuestionario de la Organización Personal en población universitaria colombiana. Informes Psicológicos, 20(1), pp. 49-62 http://dx.doi.org/10.18566/infpsic.v20n1a04 


\section{Adaptation, validation and standardization of the Mini Questionnaire of Personal Organization among Colombian universities}

\section{Abstract}

The MQPO is an Italian self-applied questionnaire to assess the Personal Meaning Organization (PM0), theoretical construct of the personality, which is fundamental for post-rationalist cognitive psychotherapy, created by Vittorio Guidano. This article is an adaptation and validation of said instrument in the Colombian population. Method: adaptation and validation were carried out by applying the instrument to a sample of 243 university students. Results: four main factors were formed as in the original version of the test, whose internal consistency indices measured using Cronbach's Alpha ranged between .57 and .67, with a total internal test consistency of .74. In the factor analysis, according to sex, a statistically significant difference was found in the Depressive PM0 ( $p=.043)$ and the Phobic PM0 $(p=.045)$, with greater tendency in men to present the former and in women the latter. Conclusion: The MQPO translated into Spanish evidenced a good internal consistency, which allows it to be used in scientific research in the Colombian university context.

\section{Adaptação, validação e estandardização do Miniquestionário da organização pessoal na população universitária colombiana}

Resumo

0 Mini Questionário da Organização Pessoal é um questionário auto aplicado de origem italiana para avaliar a organização do significado pessoal (OSP), construto teórico da personalidade fundamental para a psicoterapia cognitiva pós-racionalista, criada por Vittorio Guidano. 0 presente artigo é uma adaptação e validação deste instrumento na população colombiana. Método: a adaptação e a validação foram feitas aplicando 0 instrumento em uma amostra de 243 estudantes universitários. Resultados: conformaram-se 4 fatores principais como na versão original do teste, cujos índices de consistência interna medidos pelo Alfa de Cronbach variaram entre .57 e .67, com uma consistência interna total do teste de .74. Na análise dos fatores segundo 0 sexo achou-se uma diferença estatisticamente significativa nas OSP depressiva $(p=.043)$ e OSP fóbica $(p=.045)$, exibindo os homens uma maior tendência a expor a primeira e as mulheres a segunda organização. Conclusão: 0 Mini Questionário da Organização Pessoal traduzido ao espanhol evidenciou uma boa consistência interna. 0 que permite seu uso em pesquisas cientificas no contexto universitário colombiano..

\section{Palavras chave}

Terapia cognitiva pós-racionalista, organizações do significado pessoal, Miniquestionário da Organização Pessoal. 


\section{ntroducción}

La psicoterapia cognitiva posracionalista consiste en un método de intervención psicoterapéutica desarrollada en Italia por el psiquiatra Vittorio F. Guidano (Balbi, 1995; Moltedo, 2008; Semerari, 2002). Este método parte de las psicoterapias cognitivas estándar, pero se diferencia de ellas haciendo énfasis en la identidad personal y su evolución como sistema complejo, planteando una teoría de la personalidad y el sí mismo, u Organización del Significado Personal. Esta teoría plantea cuatro estilos de personalidad: la organización depresiva, la fóbica, obsesiva y dápica (de las siglas del italiano para trastornos alimentarios psicógenos) (Balbi, 1995; León \& Tamayo, 2011). Este es un modelo aliado al movimiento constructivista y construccionista que surge al interior de las psicoterapias cognitivas a partir de la década de los 80s del siglo pasado, en contraposición a las epistemologías racionalistas y empiristas radicales, que se fundamentan en premisas positivistas duramente cuestionadas a nivel científico ( $F i$ gueroa, Rivera, \& Maldonado, 2012)

El concepto central de la teoría del modelo posracionalista, el de Organización del Significado Personal (en adelante OSP) (Guidano, 1994), consiste en un constructo teórico que le permite al terapeuta entrenado en el método guiar el proceso de auto-observación que realiza el cliente o paciente (Balbi, 1997; Nardi \& Moltedo, 2009). Sin embargo, se hace necesario que la OSP pueda ser valorada objetivamente, de modo que se preste para ser utilizado en investigación empírica. En este sentido, Arimatea, Giovagnoli, Blasi, Bellantuono, y Nardi (2009) elaboraron un cuestionario orientado a diagnosticar la OSP llamado I/ Mini Questionario Sulla Organizzazione

Personale (MQOP) y en años recientes Nardi et al. (2012) han elaborado otro cuestionario, llamado Mini Questionnaire of Personal Organization (MQPO); éste último, redactado originalmente en italiano, fue validado en una muestra poblacional de Ancona, Italia.

En este orden de ideas, se han construido instrumentos psicométricos para evaluar el concepto teórico de OSP en investigación científica (Bertolino et al., 2005; Nardi et al., 2008; Nardi, Di Nicolò, \& Laurenzi, 2010; Picardi, Gaetano, Toni, Caroppo, \& Arciero, 2004; Picardi et al., 2003; Rubino et al., 2007). En el 2003, Picardi, Mannino, Arciero et al., realizaron el primero de estos intentos con la construcción del QSP, del italiano "questionario per la valutazione dell'organizzazione di significato personale". Para ello construyeron una versión preliminar del cuestionario basándose en aspectos teóricos clave de la teoría de Guidano para elaborar un test autoaplicado con preguntas que hacen referencia al modo de sentir y pensar de las personas sin referirse a aspectos psicopatológicos. Esta primera versión fue aplicada a 482 sujetos sanos, de los cuales 41 eran profesionales aprendices de terapia cognitiva posracionalista quienes fueron además entrevistados y diagnosticados sobre su OSP. A 167 sujetos se les aplicó la escala MMPI $L$ para medir su sesgo de deseabilidad. De esta primera prueba resultó el QSP definitivo de 68 ítems. La consistencia interna y la estabilidad del cuestionario fueron satisfactorias alfa $=.62-.82$ y un coeficiente de correlación intraclase $=.58-.84$. El análisis funcional discriminante clasificó correctamente al $75 \%$ de los aprendices utilizando los puntajes de la escala como variables independientes. Los autores utilizaron estos resultados para confirmar la hipótesis del posracionalismo de la existencia de diferentes modalidades 
de organización del significado en las personas, independientemente de su estado de normalidad o psicopatología (Picardi et al., 2003).

Posteriormente, se realizó un estudio con el propósito de probar la validez del QSP contra criterios de medición provenientes de otras formulaciones teóricas dentro del estudio de la personalidad (Picardi et al., 2004). Para lograr dicho cometido, los investigadores aplicaron a 214 sujetos sanos, además del QSP, el TCl-125, cuestionario de evaluación de personalidad según el modelo de Cloninger, el BFQ para los cinco grandes factores de personalidad, el TAS-20 de reconocimiento emocional y la escala del estilo de apego adulto ECR; adicionalmente, se aplicaron las escalas de Zung y el STAI de Spielberger para medir niveles de depresión y ansiedad. EI PMQ y las escalas STAI y Zung fueron nuevamente aplicadas a 113 sujetos tras un mes. Los resultados lanzaron una consistencia interna de QSP; alfa = .66-.82 y una confiabilidad test re-test correlación intraclase $=.70-.83$ satisfactorios . Los patrones en las puntuaciones de las diferentes escalas según las OSPs fueron ampliamente sustentados según lo hipotetizado y el diagnóstico de la OSP, el cuestionario no se vio afectado por niveles de ansiedad y depresión (Picardi et al., 2004).

Al menos tres investigaciones se han realizado utilizando el QSP como herramienta diagnóstica empírica para las OSP. La primera, cuyo título se puede traducir como "Variación en la respuesta de la amígdala humana durante estímulos amenazantes como una función del genotipo 5'HTTLPR y el estilo de personalidad" (Bertolino et al., 2005) se propone indagar la relevancia del estilo de personalidad en la variabilidad de la respuesta de la amígdala. Utilizaron dos grupos, uno Informes Psicológicos diagnosticado como poseedor de un estilo de personalidad u OSP fóbica y el otro con estilo dápico. Para su diagnóstico utilizaron el QSP complementado con entrevistas diagnósticas por profesionales expertos en el modelo. Durante un examen de resonancia magnética funcional (fMRI), a los sujetos se les aplicó una tarea perceptual de estímulos amenazantes y pareados para el genotipo del polimorfismo número variable de repeticiones en tándem 5' en el transportador de la serotonina. El estudio efectivamente diferenció estos dos tipos de personalidad según su reacción a nivel de la amígdala, los sujetos fóbicos reclutaron selectivamente la amígdala en una mayor extensión que los dápicos. La segunda investigación, titulada "La actividad en la corteza medial prefrontal durante la evaluación cognitiva de estímulos amenazantes como función del estilo de personalidad" (Rubino et al., 2007), de gran similitud a la anterior, también utiliza el QSP para discriminar entre sujetos fóbicos y dápicos en la evaluación de situaciones amenazantes. Finalmente, en el 2010 se publica el trabajo titulado "La respuesta afectiva ante el dolor de un ser amado: La actividad insular como función de diferencias individuales" (Mazzola et al., 2010), el cual consistió en nuevamente evaluar sujetos fóbicos y dápicos mediante fMRI durante el desempeño de tareas de valoración perceptual de imágenes de seres amados, esta vez evaluando el funcionamiento de la ínsula, nuevamente diferenciando empíricamente el funcionamiento neurológico de estas dos OSPs, encontrándose hay mayor actividad en los fóbicos, en esta estructura cerebral, que en los dápicos (Mazzola et al., 2010).

No obstante, el QSP no ha sido el único instrumento diagnóstico dentro del paradigma posracionalista. En 2009, Arimatea y colaboradores diseñaron un nuevo cuestionario, esta vez basado en el concepto 
del enfoque Inward/Outward inédito de Guidano, y la aproximación de las OSP de Bernardo Nardi basada en la teoría de la evolución y la adaptación (Nardi, 2008). El Mini Cuestionario de la Organización Personal fue inicialmente formulado con 40 ítems planteados por expertos terapeutas posracionalistas. Fue aplicado a una muestra de 151 sujetos, de los cuales 14 eran estudiantes de enfermería familiarizados con el modelo psicoterapéutico y a quienes se les conocía de antemano su diagnóstico de OSP. El análisis de estos 40 ítems permitió destacar que 20 de ellos eran lo suficientemente claros y discriminantes para ser utilizados en un cuestionario breve, 9 ítems más fueron modificados y los 11 restantes eliminados. Una nueva versión de 29 ítems le fue aplicada a una muestra de 160 participantes; de este segundo intento se eliminaron 9 ítems que mostraron un buen resultado de distribución y poca capacidad de discriminación entre OSPs, la versión final del mini cuestionario terminó con 20 ítems. Esta versión definitiva demostró tener índices de confiabilidad interna satisfactorios ( $\alpha=.73$ ), un acuerdo del $72.5 \%$ entre los resultados del test y la valoración clínica y del $80 \%$ entre los puntajes de este test y los del anterior PMQ (Arimatea et al., 2009; Nardi et al., 2012).

Al igual que con el $P M Q$, el MCOP también ha sido utilizado en varias investigaciones empíricas que requieren de un diagnóstico fiable que no se reduzca exclusivamente a la valoración clínica. Una investigación ha sido publicada, titulada "Activación emotiva en sujetos inward y outward: Consideraciones a la luz de un estudio de neuroimagen" (Nardi \& Capecchi, 2007); de manera similar a las investigaciones anteriormente mencionadas, utilizó el MMPI2, el QSP y el MCOP para discriminar entre sujetos con OSPs inward y outward para realizarles una tarea de reconocimiento de rostros desconocidos y sus propios rostros mientras se les practicaba un escaneo fMRI para evaluar el funcionamiento de la amígdala y otros sistemas neurológicos. Los investigadores pudieron hallar que efectivamente los sujetos Outward tienen una respuesta a la ira menos intensa y unívoca que los sujetos Inward, tienen una mayor activación cortical, no siempre responden a sus propias expresiones faciales y responden a la alegría con un mayor involucramiento del hemisferio verbal izquierdo (Nardi \& Capecchi). Estos mismos resultados fueron publicados posteriormente en español, con algunas variaciones (Nardi et al., 2008).

Los anteriores instrumentos han sido redactados en otros idiomas distintos al castellano. Ahora bien, los instrumentos mejoran sus propiedades psicométricas cuanto más se hayan usado en diferentes culturas, y con distintas poblaciones y sujetos; por esta razón, se recomienda que los instrumentos sean validados, adaptados y estandarizados con muestras que pertenezcan a la población que será evaluada en su ambiente natural (Londoño \& Alejo, 2017).

Por lo tanto, resulta necesario traducir, validar y adaptar el MCOP, puesto que en algunos países de habla hispana el posracionalismo ha encontrado un lugar particular de crecimiento, de modo que puedan realizar investigaciones científicas, tanto estudiantes y psicólogos profesionales que no tengan un entrenamiento formal en el modelo, como aquellos formados en él, facilitando el proceso de discriminación y diagnóstico de muestras de participantes en investigaciones de campo. 


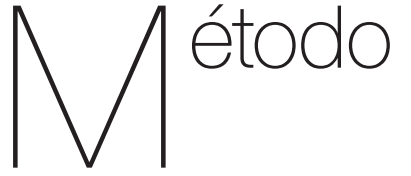

\section{Diseño}

Estudio de enfoque cuantitativo, psicométrico de corte transversal, dirigido a la adaptación, validación y estandarización de un instrumento de medición.

\section{Participantes}

Para el análisis de validez interna y confiabilidad del MCOP se incluyeron 243 estudiantes universitarios, quienes fueron seleccionados utilizando un muestreo a conveniencia. Los participantes debían cumplir como criterio de inclusión ser mayores de 18 años de edad. En cuanto a las características de los participantes, las mujeres representaron el $77.3 \%$ (153) del total de la muestra y la edad promedio en todo el grupo fue de 24.6 años (DE = 7.2). Todos los participantes pertenecían a la Institución Universitaria de Envigado, siendo estudiantes de Ciencias Sociales el 49.8\%, de Ciencias Empresariales y Derechos el 2.6\% respectivamente, y, de Ingeniería, el 9.0\%.

\section{Instrumento}

Se aplicó el Mini Cuestionario de Organización Personal (MCOP) de Nardi et al. (2012), el cual consta de 20 ítems que evalúan 4 dimensiones en la versión original (Organización obsesiva, depresiva, dápicay fóbica), en una escala tipo likert de 6 puntos; desde "no me describe nada" hasta "me describe perfectamente". Las puntuaciones altas en el cuestionario son indicativas de una predominancia de la organización personal. La Tabla
1 muestra los ítems del MCOP traducidos del italiano al español.

Tabla 1.

Ítems del MCOP traducido del italiano al español

Ítems

Para sentirme aceptado(a), es muy importante no defraudar las expectativas de los demás

Me siento asfixiado cuando no me puedo mover libremente

Es más importante para mí el esfuerzo que le dedico a mi trabajo que el reconocimiento que obtengo por él.

Para mí la soledad es la condición fundamental de la vida.

Para mí es importante saber si los demás me aceptan o no.

Me siento libre y sin restricciones cuando tengo la situación bajo control.

Para sentirme justo e imparcial, considero más mis propias reglas que la opinión de los demás.

Normalmente pienso que estoy solo(a) y que hay una gran distancia entre los demás y yo.

Para mí es importante que mi punto de vista sea compartido por las personas que valoro.

Cuando soy controlado por otros, no me siento menospreciado sino forzado y privado de mi libertad

No suelo cambiar de opinión cuando los demás me critican.

Siento que no puedo depender de nadie, por lo tanto debo comprometerme firmemente a enfrentar la realidad, ya que los resultados sólo dependen de mí.

Me siento avergonzado(a) e inadecuado(a) cuando los otros me critican o no están de acuerdo conmigo.

Para mí es esencial poder entrar y salir libremente de una situación.

Cuando se me presenta una situación de duda, las opiniones de los otros pesan menos que las mías.

Para tener éxito en la vida uno debe enfrentar el destino de la soledad.

Para mí es importante sentirme apreciado y buscado por los demás.

Me siento bien con personas de confianza, siempre y cuando no me abrumen con sus demandas.

Cuando no tengo certeza frente a una situación de duda, tiendo a ignorar las expectativas de los demás.

En mi vida siempre he tenido que arreglármelas por mi cuenta, sin poder contar con los demás. 


\section{Procedimiento}

En una primera fase del estudio, se obtuvo la autorización de los autores de la versión original del MCOP para traducir el instrumento. Luego, el cuestionario fue traducido del italiano al español por un traductor experto y certificado en Colombia, y, posteriormente se les solicitó a dos expertos con amplia experiencia en psicología clínica valorar el contenido del cuestionario. La versión del MCOP traducida al español mostró un índice de concordancia entre evaluadores en claridad del contenido de .494 .

En la segunda fase, se capacitó a cinco encuestadores en la aplicación y calificación del MCOP. El muestreo consistió en dos momentos: en el primero, cada encuestador aplicó el cuestionario a cinco personas con el fin de familiarizarse con el mismo, y en el segundo, se realizó la recolección de la información para la validación y estandarización del instrumento. A cada participante se le explicó el propósito del estudio y se le aclaró que la información suministrada se manejaría de forma confidencial. El protocolo del estudio se ajustó al código deontológico y ético del psicólogo en Colombia.

\section{Análisis de datos}

Preliminarmente, se realizó un análisis de las características psicométricas del MCOP versión en español. Para la validez interna se usó el análisis factorial exploratorio con el método de extracción de componentes principales, con rotación varimax para simplificar la interpretación de los factores. Como medidas de viabilidad para el análisis factorial se tuvo en cuenta la prueba de esfericidad de
Bartlett con un valor $\mathrm{p}<.05$, y el índice de adecuación muestral de Kaiser-MeyerOlkin $(\mathrm{KMO}) \geq$.5. Finalmente, se seleccionaron aquellos factores e ítems que los conformaban que tuvieran una confiabilidad calculada con el Alfa de Cronbach $>0.5$.

Seguidamente, los estadísticos descriptivos se obtuvieron de las puntuaciones medias totales de cada uno de los factores, esto al tener en cuenta que el número de ítems variaba en función del factor. Se comprobó la distribución normal de las variables con la prueba Kolmogorov-Smirnov, cuya hipótesis nula considera que la distribución de la variable es normal, rechazándose esta hipótesis con un valor $\mathrm{p}<.05$. El análisis de datos se realizó mediante el programa estadístico SPSS (versión 22).

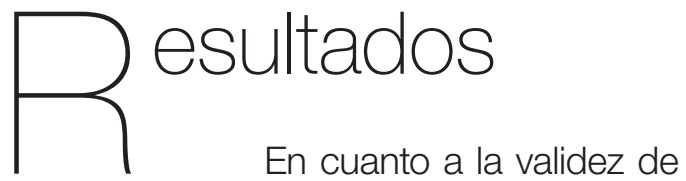
interna, los resultados de KaiserMeyer-Olkin (coeficiente $=.752$ ) y de la prueba de esfericidad de Bartlett $(\chi 2=$ 932.334, $p=.000$ ), indican que los datos de los 243 participantes iniciales eran adecuados para realizar el análisis factorial. Por lo tanto, usando el método de extracción de componentes principales con rotación varimax, se redujo a 16 reactivos de un total de 2, los cuales conformaron 4 factores que explicaron el $51.3 \%$ del total de la varianza.

La organización obsesiva quedó conformada por los ítems 7, 15 y 19. Los ítems 4, 8, 12, 16 y 20 configuraron la organización depresiva; mientras que los ítems 1, 5, 9 y 17 la organización dápica. En tanto, la organización fóbica se 
conformó con los ítems 2, 1013 y 18 (ver Tabla 2). Cada uno de estos factores explicó, respectivamente, el 13.37\%, 13.23\%, $13.38 \%$, y $11.33 \%$ de la varianza total. Los ítems 3, 6, 11 y 14 fueron eliminados dado que conformaban factores que no cumplían con el criterio establecido en el estudio. El ítem 13: "me siento avergonzado(a) e inadecuado(a) cuando los otros me critican o no están de acuerdo conmigo", en la versión original pertenecía a la organización dápica, mientras que en el estudio actual se incluyó por análisis factorial en la organización fóbica, los otros ítems no mostraron cambios en comparación con la versión original.

Tabla 2

Factores de la versión en español del MCOP

\begin{tabular}{|c|c|c|c|c|}
\hline \multirow{2}{*}{ Número de ítem } & \multicolumn{4}{|c|}{ Factores } \\
\hline & 1 & 2 & 3 & 4 \\
\hline ítem 19 & .823 & & & \\
\hline ítem 15 & .741 & & & \\
\hline ítem 7 & .590 & & & \\
\hline ítem 16 & & .695 & & \\
\hline ítem 8 & & .679 & & \\
\hline ítem 12 & & .653 & & \\
\hline ítem 4 & & .575 & & \\
\hline ítem 20 & & .495 & & \\
\hline ítem 17 & & & .728 & \\
\hline ítem 1 & & & .678 & \\
\hline ítem 5 & & & .666 & \\
\hline ítem 9 & & & .636 & \\
\hline ítem 10 & & & & .737 \\
\hline ítem 18 & & & & .644 \\
\hline ítem 2 & & & & .596 \\
\hline ítem 13 & & & & .397 \\
\hline
\end{tabular}

La confiabilidad, calculando el Alfa de Cronbach para la escala total, teniendo en cuenta que algunos ítems fueron eliminados por el análisis factorial, fue de .74 por lo que se considerada que el MCOP traducido al español tiene una consistencia interna adecuada. En las dimensiones se encontraron valores de Alfa de Cronbach aceptables, siendo el más bajo el de la organización fóbica (ver Tabla 3).

Tabla 3.

Confiabilidad para cada factor del MCPO versión en español

\begin{tabular}{ll}
\hline OSP & $\alpha$ \\
Obsesiva & .67 \\
Depresiva & .64 \\
Dápica & .65 \\
Fóbica & .57 \\
Total del cuestionario & .74 \\
\hline
\end{tabular}

Nota: $\mathrm{OSP}=$ Organización del significado personal.

En la Tabla 4 se muestran los valores estadísticos de las medidas de descriptivas de los factores del MCOP. Se puede evidenciar que en la muestra el promedio más alto fue el de la organización fóbica seguida por la dápica, lo que igualmente corresponde con el 50\% de la muestra en el análisis de percentiles. Según los coeficientes de asimetría, la única organización con tendencia a distribuir normal es la dápica, lo que se confirma con la prueba de Kolmogorov-Smirnov ( $p=$.065).

Tabla 4.

Estadísticos descriptivos de los factores que conforman el MCOP versión en español

\begin{tabular}{lccccc}
\hline Factores & Media (DE) & P25 & P50 & P75 & $\begin{array}{c}\text { Coeficiente } \\
\text { de asimetría }\end{array}$ \\
\hline Obsesiva & $\begin{array}{c}2.49 \\
(1.07)\end{array}$ & 1.66 & 2.33 & 3.33 & .630 \\
Depresiva & $\begin{array}{c}3.00 \\
(1.05)\end{array}$ & 2.20 & 3.00 & 3.60 & .174 \\
Fóbica & $\begin{array}{c}3.51 \\
(.97)\end{array}$ & 3.00 & 3.50 & 4.25 & -.417 \\
Dápica & $\begin{array}{c}3.23 \\
(.98)\end{array}$ & 2.50 & 3.25 & 4.00 & .003 \\
\hline
\end{tabular}

Nota: DE: Desviación estándar, $\mathrm{P}=$ Percentil 
Se compararon cada uno de los factores del MCOP según el sexo, mostrando los hombres una mayor tendencia a las organizaciones obsesiva y depresiva, mientras que las mujeres a las organizaciones fóbica y dápica, sin embargo, se encontraron únicamente diferencias estadísticamente significativas entre los hombres y las mujeres en la organización depresiva y fóbica.

Tabla 5.

Comparación de los factores que conforman el MCOP según el sexo

\begin{tabular}{lccc}
\hline Factores & Hombres & Mujeres & $\begin{array}{c}\text { Nivel de } \\
\text { significancia. }\end{array}$ \\
\hline Obsesiva & $2.66(1.67)$ & $2.33(1.33)$ & .071 \\
Depresiva & $\begin{array}{c}3.18 \\
(1.80)\end{array}$ & $2.89(1.40)$ & $.043^{\star}$ \\
Fóbica & $\begin{array}{l}3.50 \\
(1.06)\end{array}$ & $3.75(1.25)$ & $.045^{\star}$ \\
Dápica & 3.20 & $3.25(.95)$ & .673 \\
\hline
\end{tabular}

Nota: OPS obsesiva, depresiva y fóbica = mediana (Rango Intercuartil), prueba U de Mann Whitney. Organización dápi$\mathrm{ca}=$ media (desviación estándar), prueba $\mathrm{t}$ de student. ${ }^{*} \mathrm{p}<.05$

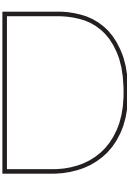

\section{iscusión}

En concordancia con el cuestionario original en italiano, elaborado por Nardi et al. (2012), el cuestionario adaptado y validado continúa siendo coherente con el modelo adaptativo posracionalista que propone el mismo Nardi (Nardi, 2007; Nardi \& Bellantuono, 2008; Nardi, Bellantuono, \& Moltedo, 2010). Éste consiste en una re-evaluación del modelo de personalidad u Organización del Significado Personal (OSP) que propuso Guidano, partiendo de su obra seminal junto con Giovanni Liotti publicada en 1983 (Guidano \& Liotti, 2006). Es decir, que la presente adaptación y validación del cuestionario de Nardi le aporta soporte empírico al constructo teórico del modelo posracionalista propuesto por Guidano y sus cuatro organizaciones del significado personal.

En esta investigación la confiabilidad del instrumento medida por el Alfa de Cronbach fue similar a la obtenida en la investigación de Nardi (2012), obteniéndose un $\alpha=.74$. Este resultado puede explicarse dado que las OSP constituyen categorías de personalidad que no dependen única y exclusivamente de los contextos culturales.

Por otro lado, la varianza total explicada por los cuatro factores, correspondientes a las cuatro OSPs, obtenida en la presente validación es del 51.3\%, mientras que la obtenida por Nardi et al. (2012) fue de $42.2 \%$, lo que está indicando que pueden existir otras organizaciones que aún no se han conceptualizado. Guidano (1987; 1994; 2001; 2002; 2013) por su parte, aclaró en varias fuentes que podría considerarse la posibilidad de proponer otras organizaciones de la personalidad, aunque probablemente no serían demasiadas, ya que las emociones básicas que posibilitan el cierre organizacional sobre el que se fundamenta un sentido de continuidad de sí mismo son pocas. Es decir, ya que el sentido de sí mismo se organiza emocionalmente durante el desarrollo, y debido a que las emociones básicas de las que dispone un ser humano desde la infancia son pocas, las posibilidades emocionales sobre las cuales la personalidad se puede comenzar a formar son también limitadas. Éste es un argumento puramente hipotético de Guidano, sin embargo, Arciero, por su parte, ha propuesto la existencia de una quinta organización o estilo de la personalidad, al que ha llamado histérica-hipocondriaca (Arciero \& Bondolfi, 2009). 
Analizando los reactivos, se encontró que el número 13 del cuestionario: "me siento avergonzado(a) e incómodo(a) cuando los otros me critican o no están de acuerdo conmigo", perteneciente en la versión original al factor correspondiente a la OSP Dápica, pasó a formar parte del factor correspondiente a la OSP Fóbica. Esto podría deberse a que la muestra seleccionada tiene una edad promedio de 24.6 años, es decir, es una muestra compuesta de universitarios jóvenes en su mayoría. Guidano (1987; 1994) describió la OSP Dápica como una organización de la personalidad en donde el sentido de sí mismo es difuso y ambiguo para el propio sujeto, logrando una estabilidad en el sentido personal a partir de cómo se ve percibido por los otros. Este modo de funcionamiento es también característico de los adolescentes y jóvenes adultos en general, independientemente de su organización o tipo de personalidad (Arciero \& Bondolfi, 2009; Balbi, 2011; 2015); es factible hipotetizar que un ítem o reactivo propio de la OSP Dápica puntúe alto en otras organizaciones debido a que los participantes son jóvenes y tienen una inclinación a operar de modo semejante a alguien de OSP Dápica en algunas instancias, particularmente en lo referente a la sensibilidad al juicio externo, especialmente de pares. Además, a diferencia de las personas típicas de OSPs Depresiva y Obsesiva, las personas de OSP Fóbica tienen una inclinación a ser más socialmente abiertos, focalizados sobre el distanciamiento óptimo con respecto a figuras significativas que provean seguridad, al tiempo que permitan un margen de libertad; mientras que las otras organizaciones pueden tender más a la evitación interpersonal (e.g. en el caso de la OSP Depresiva), o bien a la relativa indiferencia interpersonal (e.g. en el caso de la OSP
Obsesiva) (Guidano, 2002; Nardi, 2007; 2008; Nardi \& Moltedo, 2008; Oneto \& Moltedo, 2002).

Los resultados obtenidos demostraron medias más altas en la muestra de las OSPs Fóbica y Dápica con respecto a las otras dos organizaciones restantes. Este dato podría deberse a que, en términos de su desarrollo ontogénico, la OSPs Fóbica se comienza a constituir a partir de un patrón de apego ambivalente durante la infancia, el cual es consistente durante la historia del desarrollo evolutivo del individuo en interacción con sus vínculos significativos, es factible suponer que podrían existir cualidades culturales regionales que promuevan una mayor prevalencia de este tipo de patrones de apego. Con respecto a la OSP Dápica, esta se observará como un estilo de personalidad propio de la adolescencia y la adultez temprana, incluso en ocasiones en las que podría el individuo pertenecer a otra OSP (Balbi, 2015), por lo tanto, este dato podría deberse a las características demográficas universitarias de la muestra poblacional. Por otra parte, las organizaciones Depresiva y Obsesiva, son organizaciones de la personalidad que, en la experiencia clínica, se observan más escasos que las otras dos, lo cual ha sido observado desde los inicios de las bases teóricas del concepto de OSP (Guidano \& Liotti, 2006). Esto se debe a que, en condiciones socioeconómicas y demográficas estables, más bien pacíficas, provechosas y con oportunidad de crecimiento, los patrones de apego evitantes, que constituyen el punto de inicio de desarrollo de la OSP Depresiva, tendrán una menor prevalencia, puesto que éstos proliferan en condiciones hogareñas adversas (Crittenden, 1992). Similarmente, se observa en las práctica clínica una 
menor prevalencia de la OSP Obsesiva (Guidano \& Liotti, 2006), pues su desarrollo es más complicado y requiere de una historia de reciprocidad con los vínculos afectivos durante el desarrollo centrados en la función pedagógica y verbal (Nardi, Bellantuono et al., 2010), que podría ser menos idiosincrático de la cultura local.

Finalmente, se halló que en la muestra seleccionada hay más hombres de OSP Depresiva y más mujeres con OSP Fóbica, mientras que las diferencias en cuanto al sexo en las otras dos organizaciones no resultó estadísticamente significativa. Este hallazgo puede deberse a prácticas o patrones de crianza culturalmente transmitidas a nivel regional, patrones de apego ansioso podrían ser más frecuentes en mujeres y patrones evitantes en hombres localmente, lo cual podría conllevar a que se consoliden más mujeres adultas de OSP Fóbica y hombres de OSP Depresiva. Este hallazgo se encuentra en consonancia con los resultados obtenidos inicialmente por los estudios clínicos seminales de Guidano y Liotti (1983/2006) a partir del cual propusieron la existencia de cuatro organizaciones de la personalidad, en aquel entonces llamadas "estructuras cognitivas". Este estudio se hizo con una muestra de 198 y otro estudio previo (Liotti, 1985) en el cual hallaron una mayor prevalencia de la estructura cognitiva fóbica (precursor conceptual de la OSP fóbica) en mujeres y de la estructura cognitiva depresiva en hombres.

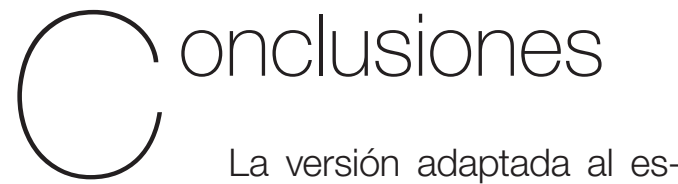
pañol del MCOP y su validación en población colombiana ha obtenido el mismo grado de consistencia interna que la versión original desarrollada por Nardi et al. (2012) en italiano.

Los resultados obtenidos en la presente investigación validan el modelo de las organizaciones de significado personal, pertenecientes al marco teórico de la psicoterapia cognitiva posracionalista, creada por Vittorio Guidano y de la actual conceptualización por Bernardo Nardi, creador del instrumento original, en la población colombiana a la cual aplica la adaptación y validación del instrumento. Esta información evidencia, además, que las OSP no dependen exclusivamente de la cultura, en cuanto se ha hallado esta información tanto en población italiana como en colombiana.

Al interior de la muestra poblacional elegida para la aplicación del instrumento adaptado, se pudo hallar diferencias en función del sexo: Una mayor presencia de la OSP Depresiva en los hombres y de OSP Fóbica en las mujeres. Contando con el instrumento adaptado y validado en población local, tendría valor realizar investigaciones que busquen correlacionar aspectos relevantes de la cultura local que puedan tener una influencia en el apego y en las OSP de las personas pertenecientes a dicha cultura.

Finalmente, a pesar del soporte empírico que la presente investigación proporciona al constructo teórico de las Organizaciones de Significado Personal, debe tenerse en cuenta que éste es un constructo diseñado para servir como heurístico que guía el ejercicio clínico de psicoterapeutas entrenados en el método y en el modelo teórico de la terapia cognitiva posracionalista. Con esto se quiere decir que la versión adaptada y validada del MCOP debe utilizarse con fines 
y en contextos netamente académicos e investigativos, y no debe usarse como instrumento psicométrico en la práctica clínica, puesto que el instrumento en sí no puede reemplazar el juicio clínico de un psicoterapeuta experto entrenado formalmente en el modelo.

\section{Limitaciones}

Dado que el instrumento fue adaptado y validado en población universitaria, compuesta principalmente por jóvenes, las puntuaciones de referencia no son aplicables a otro tipo de poblaciones.

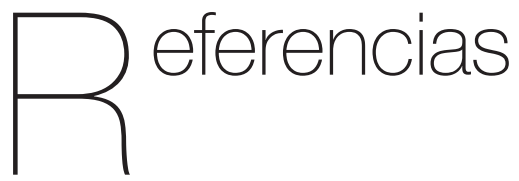

Arciero, G., \& Bondolfi, G. (2009). Selfhood, Identity and Personality Styles. Chichester: Wiley and Sons.

Arimatea, E., Giovagnoli, S., Blasi, S., Bellantuono, C., \& Nardi, B. (2009). ॥ mini questionario sulla organizzazione personale (MQOP): Studio preliminare di validazione. Quaderni di Psicoterapia Cognitiva 25, 14(2), 178-201.

Balbi, J. (1995). Vittorio Guidano y el enfoque cognitivo post-racionalista. Notas sobre una entrevista. Revista de Psicoterapia, 6(24), 91. Recuperado de http://revistadepsicoterapia.com/vittorioguidano-y-el-enfoque-cognitivo-postracionalista-notas-sobre-una-entrevista. html
Balbi, J. (1997). El proceso terapéutico en la terapia cognitiva posracionalista. In I. Caro (Ed.), Manual de Psicoterapias Cognitivas (pp. 381-398). Barcelona: Paidós.

Balbi, J. (2011). Metarappresentazione affettiva tacita e senso di identità personale. Un approccio alla comprensione delle gravi patologie psichiatriche dell'adolescenza e giovinezza. Rivista di Psichiatria, 46(5-6), 337-342.

Balbi, J. (2015). Adolescence, order through fluctuations and psychopathology. A postrationalist conception of mental disorders and their treatment on the grounds of chaos theory. Chaos and Complexity Letters, 9(2), 85-105.

Bertolino, A., Arciero, G., Rubino, V., Latorre, V., De Candia, M., Mazzola, V., ... Scarabino, T. (2005). Variation of human amygdala response during threatening stimuli as a function of 5'HTTLPR genotype and personality style. Biological Psychiatry, 57(12), 1517-1525.

Crittenden, P. M. (1992). Children's strategies for coping with adverse home environments: An interpretation using attachment theory. Child Abuse \& Neglect, 16(3), 329-343. https://doi. org/10.1016/0145-2134(92)90043-Q

Figueroa, J., Rivera, C., \& Maldonado, N. (2012). Positivismo vs. construccionismo: Implicaciones en la práctica del psicodiagnóstico y la psicoterapia. Informes Psicológicos, 12(2), 11-27. Recuperado de https://revistas.upb.edu. co/index.php/informespsicologicos/article/ view/1717/1830

Guidano, V. F. (1987). Complexity of the self: A developmental approach to 
psychopathology and therapy. Nueva York: Guilford Press.

Guidano, V. F. (1994). El sí mismo en proceso: Hacia una terapia cognitiva posracionalista. Barcelona: Paidós.

Guidano, V. F. (2001). Vittorio Guidano en Chile. (S. Aronsohn, Ed.). Santiago de Chile: Universidad Academia de Humanismo Cristiano.

Guidano, V. F. (2002). El modelo cognitivo postracionalista: Hacia una reconceptualización teórica y clínica. Barcelona: Desclée de Brouwer.

Guidano, V. F. (2013). La psicoterapia entre arte y ciencia. Revista de Psicoterapia, 24(9496), 11-236.

Guidano, V. F., \& Liotti, G. (2006). Procesos cognitivos y desórdenes emocionales. Santiago de Chile: Cuatro Vientos.

León, A., \& Tamayo, D. A. (2011). Lapsicoterapia cognitiva posracionalista: un modelo de intervención centrado en el proceso de construcción de la identidad. Katharsis, (12), 37-58. Recuperado de http://revistas. iue.edu.co/index.php/katharsis/article/ view/153

Liotti, G. (1985). Un modello cognitivocomportamentale per l'agorafobia. In V. F. Guidano \& M. Reda (Eds.), Cognitivismo e psicoterapia (pp. 149-150). Milan: Franco Angeli.

Londoño, C, \& Alejo, I. (2017). Instrumentos usados en Colombia para evaluar la dimensión psicológica del proceso salud-enfermedad. Bogotá: Universidad Católica de Colombia.

Mazzola, V., Latorre, V., Petito, A., Gentili, N., Fazio, L., Popolizio, T., ... Bondolfi, G. (2010). Affective response to a loved one's pain: Insula activity as a function of individual differences. PloS One, 5(12), e15268. Retrieved from https://doi.org/10.1371/ journal.pone.0015268

Moltedo, A. (2008). La evolución de la obra y el modelo de Vittorio Guidano: Notas histórico biográficas. Revista de Psicología, 18(1), 65-86.

Nardi, B. (2007). Costruirsi: Sviluppo e adattamento del sé nella normalità e nella patologia. Milán: FrancoAngeli.

Nardi, B. (2008). La organización del sí mismo: De la derivación psicopatológica de Guidano al significado adaptativo de construcción de la reciprocidad. Revista de Psicoterapia, 19(74/75), 103-131.

Nardi, B., Arimatea, E., Giovagnoli, S., Blasi, S., Bellantuono, C., \& Rezzonico, G. (2012). The mini questionnaire of personal organization (MQPO): Preliminary validation of a new post-rationalist personality questionnaire. Clinical Psychology and Psychotherapy, 1-13. Retrieved from http://onlinelibrary. wiley.com/doi/10.1002/cpp.740/full

Nardi, B., \& Bellantuono, C. (2008). A new adaptive and evolutionary conceptualization of the Personal Meaning Organization (PMO) framework. European Psychotherapy, 8(1), 5-16. 
Nardi, B., Bellantuono, C., \& Moltedo, A. (2010). Una aproximación adaptativo-evolutiva a las organizaciones de significado personal de Vittorio Guidano: Nuevas perspectivas en la psicoterapia post-racionalista. Gaceta de Psiquiatría Universitaria, 6(1), 63-70. Recuperado de http://www. gacetadepsiquiatriauniversitaria.cl/ ediciones/a6vol6n1mar2010/pdf/GPU 2010-1.pdf\#page=63

Nardi, B., \& Capecchi, I. (2007). Attivazioni emotive in soggetti inward e outward: Considerazioni alla luce di una indagine di neuroimaging. Quaderni di Psicoterapia Cognitiva 21(12/2), 8-24.

Nardi, B., Capecci, I., Fabri, M., Polonara, G., Salvolini, U., Bellantuono, C., \& Moltedo, A. (2008). Estudio mediante imagen funcional de resonancia magnética (fMRl) de las activaciones emotivas correlacionadas a la presentación de rostros extraños o del propio rostro en sujetos con personalidad inward y outward. Revista Chilena de Neuro-Psiquiatría, 46(3), 168-181. Recuperado de http:// www.scielo.cl/scielo.php?pid=S0717$92272008000300002 \&$ script $=$ sci_ arttext\&tlng=pt

Nardi, B., Di Nicolò, M., \& Laurenzi, S. (2010). L'emergere della coscienza. Prima parte: La scoperta di sé. In Forum Multiprofessionali di Scienze Umane (pp. 18-22).

Nardi, B., \& Moltedo, A. (2008). Rol de la relación de reciprocidad en el desarrollo de las diversas organizaciones de significado personal. Gaceta de Psiquiatría Universitaria, 4(3), 345-358.
Nardi, B., \& Moltedo, A. (2009). Desarrollo del modelo de las organizaciones de significado personal de Vittorio Guidano: Los procesos de construcción del sí en el modelo cognitivo post-racionalista. Psicoperspectivas, 8(1), 93-111.

Oneto, L., \& Moltedo, A. (2002). Las Organizaciones de Significado Personal de Vittorio Guidano: Una llave explicativa de la experiencia humana. Psicoperspectivas, 1(1), 83-92.

Picardi, A., Gaetano, P., Toni, A., Caroppo, E., \& Arciero, G. (2004). Sostegno alla teoria delle "organizzazioni di significato personale" da altre elaborazioni teoriche nell'area della personalità: uno studio di validità convergente del QSP. Rivista di Psichiatria, 39, 112-124.

Picardi, A., Mannino, G., Arciero, G., Gaetano, P., Pilleri, M. F., Arduini, L., ... Reda, M. A. (2003). Costruzione e validazione del QSP, uno strumento per la valutazione dello stile di personalità secondo la teoria delle "organizzazioni di significato personale." Revista di Psichiatria, 38(1), 13-34.

Rubino, V., Blasi, G., Latorre, V., Fazio, L., D'Errico, I., Mazzola, V., ... Bertolino, A. (2007). Activity in medial prefrontal cortex during cognitive evaluation of threatening stimuli as a function of personality style. Brain Research Bulletin, 74(4), 250-257.

Semerari, A. (2002). Historia, teorías y técnicas de la psicoterapia cognitiva. Barcelona: Paidós. 